\title{
Correction to: Structurally Diverse Polymethylated Phloroglucinol Meroterpenoids from Baeckea frutescens
}

\author{
Yin-E Zhi ${ }^{1} \cdot$ Xu-Jie Qin ${ }^{1} \cdot$ Hui Liu ${ }^{1} \cdot$ Yuan Zeng ${ }^{1} \cdot$ Wei Ni $^{1} \cdot$ Li He $^{2} \cdot$ Zu-Ding Wang ${ }^{3} \cdot$ Hai-Yang Liu ${ }^{1}(0)$
}

Published online: 24 December 2018

(c) The Author(s) 2018

\section{Correction to:}

Natural Products and Bioprospecting (2018) 8:431-439

https://doi.org/10.1007/s13659-018-0189-3

In the original publication, two errors have occurred. The corrected texts are provided below:

1. The author, Xu-Jie Qi, should read as Xu-Jie Qin among the author group.

2. The reference 9 should be cited as X.J. Qin, Y.E. Zhi, H. Yan, Y. Zhang, H. Liu, Q. Yu, S. Wang, Q. Zhao, L. He, X. Ma, L.K. An, H.Y. Liu, Tetrahedron 74, 6658-6666 (2018).

\begin{abstract}
Open Access This article is distributed under the terms of the Creative Commons Attribution 4.0 International License (http://creativecommons.org/licenses/by/4.0/), which permits unrestricted use, distribution, and reproduction in any medium, provided you give appropriate credit to the original author(s) and the source, provide a link to the Creative Commons license, and indicate if changes were made.
\end{abstract}

The original article can be found online at https://doi.org/10.1007/ s13659-018-0189-3.

\section{Hai-Yang Liu}

haiyangliu@mail.kib.ac.cn

1 State Key Laboratory of Phytochemistry and Plant Resources in West China, Kunming Institute of Botany, Chinese Academy of Sciences, and Yunnan Key Laboratory of Medicinal Chemistry, Kunming 650201, China

2 Department of Dermatology, The First Affiliated Hospital of Kunming Medical University, Kunming 650032, China

3 Kunming Botanee Bio-Technique Co. Ltd, Kunming 650106, China 